\title{
A To-Do List at Diagnosis of Systemic Sclerosis with Positive Anti-RNA Polymerase III Antibodies
}

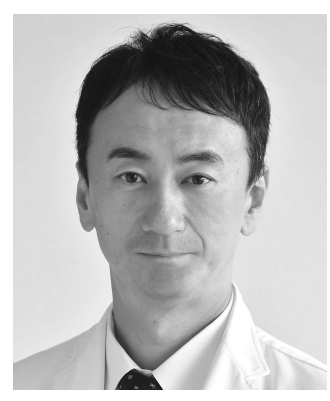

The presence of circulating antinuclear antibodies (ANA) is one of the distinctive features of systemic sclerosis (SSc), or scleroderma. A variety of SSc-related ANA, including anticentromere antibody (ACA), antitopoisomerase I antibody (anti-topo I), and anti-RNA polymerase (RNAP) III antibody, have been well characterized and are used in our daily clinical practice. They are specific to SSc and are associated with unique sets of disease manifestations. Because of their mutual exclusiveness, SSc-related ANA are widely used for diagnosis, designation of clinical subsets, and prediction of future organ involvement and prognosis ${ }^{1}$.

Serum autoantibodies reactive with multiple RNAP specificities, including RNAP III, were first identified by our group in 1993 using an immunoprecipitation assay ${ }^{2}$. We reported anti-RNAP III antibody as a biomarker that is highly specific to SSc and is associated with diffuse cutaneous SSc $(\mathrm{dcSSc})$ and scleroderma renal crisis (SRC). After our initial report, these clinical correlations were confirmed by several independent studies, but anti-RNAP III antibody measurement was not used in clinical practice because an immunoprecipitation assay, which requires complicated procedures with a radioisotope and cultured human cell line, was the only method for detection. Because eukaryotic RNAP III consists of at least 13 subunits, identification of antigenic epitope(s) recognized by all anti-RNAP III-positive sera is necessary for development of convenient immunoassays. To overcome this limitation, we conducted a series of experiments using individual subunits and their recombinant fragments, and successfully identified the central portion of the largest subunit RPC155/POLR3A as the major antigenic site universally recognized by anti-RNAP III-positive SSc sera ${ }^{3,4}$. Use of the recombinant antigenic fragment enabled us to establish a sensitive and specific immunoassay system ${ }^{4}$. Currently, commercial immunoassay kits using this system (MBL, INOVA, and Phadia) are available as in vitro diagnostics in many countries. This evolution has significantly contributed to inclusion of anti-RNAP III antibody in new American College of Rheumatology/European League Against
Rheumatism criteria for classification of SSc, based on broad consensus among experts about the utility of anti-RNAP III antibody in classifying $\mathrm{SSc}^{5}$.

The spread of commercial anti-RNAP III antibody assays has enormously promoted a number of clinical studies assessing clinical correlations of anti-RNAP III antibodies. All studies conducted in various countries and ethnic groups have confirmed strong associations of anti-RNAP III antibodies with dcSSc and SRC. In particular, within dcSSc population, anti-RNAP III antibody is associated with rapidly progressive skin thickening and a shorter interval between the appearance of the first SSc-related symptoms and the peak of skin thickness ${ }^{6}$. It is now obvious that patients with anti-RNAP III antibody have the highest risk for developing SRC, but they seldom develop severe interstitial lung disease. In patients with SSc who are anti-RNAP III-positive, prevalence of SRC during the disease course ranges from $14 \%$ to $51 \%$. In Northern Europe and North America, about $60 \%$ of patients experiencing SRC carry anti-RNAP III antibody ${ }^{7}$. Lower incidence of SRC in Japan, France, and Mediterranean countries is thought to reflect the lower prevalence of anti-RNAP III antibody within these populations when compared with the UK and North America ${ }^{8}$. Despite the lower prevalence of anti-RNAP III antibody in French and Japanese SSc patients, this antibody remains the strongest serological marker for $\mathrm{SRC}^{8,9}$. On the other hand, analysis involving a large number of patients revealed clinical features potentially associated with anti-RNAP III antibodies, which were previously unnoticed. These included gastric antral vascular ectasia (GAVE; or "watermelon stomach") ${ }^{10}$ and pulmonary hypertension ${ }^{11}$.

In addition, it has been highlighted that there is an association with cancer among SSc patients with anti-RNAP III antibodies in close temporal relationship to SSc onset ${ }^{12,13}$. It has long been recognized that patients with SSc have an increased age- and sex-adjusted risk of cancer compared to the general population ${ }^{14}$. This increased risk could be secondary to damage from the disease process itself (i.e.,

See Malignancies in anti-RNAP3+ SSc, page 639

Personal non-commercial use only. The Journal of Rheumatology Copyright @ 2017 . All rights reserved. 
lung cancer), or a consequence of the immunosuppressive therapies used to treat SSc (i.e., hematologic malignancies), although there is a subset of patients with SSc diagnosed with cancer within a short interval of the first clinical signs of $\mathrm{SSc}^{15}$; the majority of them have breast cancer ${ }^{16}$. Despite strong associations of SSc-related ANA with a variety of clinical characteristics, the correlation with cancer had never been mentioned. In fact, Shah, et al reported that the prevalence of cancer was almost similar among SSc patients with anti-RNAP III, ACA, and anti-topo $\mathrm{I}^{12}$. In contrast, the median disease duration at cancer diagnosis was quite different: 1.2 years in anti-RNAP III-positive patients, in contrast to 13.4 and 11.1 years in anti-topo I or ACA-positive patients, respectively ${ }^{12}$. The temporal relationship between cancer and SSc among anti-RNAP III-positive patients suggests that $\mathrm{SSc}$ is a paraneoplastic disorder in this patient subset ${ }^{17}$. This theory is further supported by the presence of missense mutations in the RPC155/POLR3A gene in the cancer tissue and mutation-specific T cell responses in SSc patients with anti-RNAP III antibodies, but not in those with anti-topo I or $\mathrm{ACA}^{18}$.

In this issue of The Journal, Lazzaroni, et al examined characteristics of anti-RNAP III-positive patients with SSc using the European Scleroderma Trials and Research (EUSTAR) registry, the largest multicenter cohort of patients with $\mathrm{SSc}^{19}$. Especially, a case-control study strategy involving anti-RNAP III-positive and -negative patients with SSc matched for sex, disease duration, disease subset, and age at disease onset was useful in eliminating potential influences of confounding factors. As expected, multivariate analysis identified SRC, GAVE, rapid progression of skin thickness, and malignancies concomitant to SSc onset as independent characteristics associated with anti-RNAP III antibodies, although the association with pulmonary hypertension was not confirmed. In addition, Lazzaroni's study has provided valuable information on cancer development in patients with SSc who have anti-RNAP III antibodies, including that (1) the majority of cancer was diagnosed within an interval between 6 months before and 12 months after SSc onset; (2) the most prevalent cancer type was breast cancer, (3) patients with older age or dcSSc were particularly at risk, and (4) malignancies other than breast cancer were much more frequent in males ${ }^{19}$. Nevertheless, frequency of cancer diagnosis within 2 years before or after SSc onset in anti-RNAP III-positive patients was only $11 \%$, and the number of anti-RNAP III-positive patients needed to screen to find 1 synchronous cancer at SSc diagnosis was about 17. This is much lower than dermatomyositis patients with anti-transcriptional intermediary factor (TIF) $1-\gamma$ antibodies, another autoantibody associated with paraneoplastic connective tissue disease. A metaanalysis showed that the prevalence of cancer diagnosis within 2 years before or after dermatomyositis onset in anti-TIF1 $\gamma$-positive patients was $79 \%{ }^{20}$.

Because patients with anti-RNAP III antibodies have a significantly increased risk of cancer within a short interval of SSc onset, these patients are likely to benefit from rigorous cancer screening at the time of diagnosis. However, the optimal approach for cancer screening in such patients remains unknown. In this regard, we have to appreciate a relatively low prevalence of concomitant cancer $(\sim 10 \%)$ at diagnosis in anti-RNAP III-positive patients with SSc, and consider invasiveness and radiation exposure of screening tests as well as economic issues. In this case, cancer risks identified by Lazzaroni's study may help in clinical practice to institute appropriate cancer screening ${ }^{19}$. Specifically, recommendations for screening of synchronous cancer in anti-RNAP III-positive patients with SSc are proposed using a Delphi exercise by the EUSTAR experts ${ }^{19}$. These include screening for breast cancer in all female patients, and non-invasive tests guided by clinical suspicion and patient age for other malignancies for all patients. However, some degree of discordance among the experts was found on the use of whole-body positron emission tomography/computerized tomography and duration of tight followup surveillance. At present, there is general consensus that careful screening of synchronous cancer is recommended in all patients when anti-RNAP III-positive SSc is diagnosed, but screening tests required and the length of the followup period should be decided based on the individual patient's risk.

The application of circulating SSc-related ANA measurement to clinical practice has greatly improved our patient care. Indeed, availability of convenient anti-RNAP III antibody testing has brought drastic changes in the management of SSc patients. The presence of anti-RNAP III antibody at SSc diagnosis should alert the managing physician to increased risk of developing rapidly progressing skin thickness and SRC as well as the presence of synchronous malignancy. Close monitoring of skin manifestations, such as semiquantitative skin thickness scoring, degree of joint contractures, and flexion tendon rubs, is essential because early progressive dcSSc is an ideal indication for aggressive disease-modifying therapies. In addition, appropriate monitoring of blood pressure for early detection of SRC is mandatory because the prompt introduction of an angiotensin-converting enzyme inhibitor can be life-saving. When an anti-RNAP III antibody test comes back with a positive result in patients with new-onset $\mathrm{SSc}$, we have to perform many investigations, including careful monitoring of skin and renal manifestations as well as prompt malignancy screening. These efforts will surely contribute to further improvement of outcomes in this subset of patients with SSc.

\section{MASATAKA KUWANA, $\mathrm{MD}, \mathrm{PhD}$, Department of Allergy and Rheumatology, Nippon Medical School Graduate School of Medicine, 1-1-5 Sendagi, Bunkyo-ku, Tokyo 113-8602, Japan.}

Address correspondence to Prof. M. Kuwana; E-mail: kuwanam@nms.ac.jp Personal non-commercial use only. The Journal of Rheumatology Copyright (c) 2017. All rights reserved. 


\section{REFERENCES}

1. Kuwana M. Circulating anti-nuclear antibodies in systemic sclerosis: utility in diagnosis and disease subsetting. J Nippon Med Sch 2017; In press.

2. Kuwana M, Kaburaki J, Mimori T, Tojo T, Homma M. Autoantibody reactive with three classes of RNA polymerases in sera from patients with systemic sclerosis. J Clin Invest 1993;91:1399-404

3. Kuwana M, Okano Y, Kaburaki J, Medsger TA Jr, Wright TM. Autoantibodies to RNA polymerases recognize multiple subunits and demonstrate cross-reactivity with RNA polymerase complexes. Arthritis Rheum 1999;42:275-84.

4. Kuwana M, Kimura K, Kawakami Y. Identification of an immunodominant epitope on RNA polymerase III recognized by systemic sclerosis sera: application to enzyme-linked immunosorbent assay. Arthritis Rheum 2002;46:2742-7.

5. van den Hoogen F, Khanna D, Fransen J, Johnson SR, Baron M, Tyndall A, et al. 2013 classification criteria for systemic sclerosis: an American College of Rheumatology/European League against Rheumatism collaborative initiative. Arthritis Rheum 2013; 65:2734-47.

6. Wirz EG, Jaeger VK, Allanore Y, Riemekasten G, Hachulla E, Distler $\mathrm{O}$, et al. Incidence and predictors of cutaneous manifestations during the early course of systemic sclerosis: a 10-year longitudinal study from the EUSTAR database. Ann Rheum Dis 2016;75:1285-92.

7. Penn H, Howie AJ, Kingdon EJ, Bunn CC, Stratton RJ, Black CM, et al. Scleroderma renal crisis: patient characteristics and long-term outcomes. QJM 2007;100:485-94.

8. Sobanski V, Dauchet L, Lefèvre G, Lambert M, Morell-Dubois S, Sy T, et al. Prevalence of anti-RNA polymerase III antibodies in systemic sclerosis: new data from a French cohort and a systematic review and meta-analysis. Arthritis Rheumatol 2014;66:407-17.

9. Kuwana M, Kaburaki J, Okano Y, Tojo T, Homma M. Clinical and prognostic associations based on serum antinuclear antibodies in Japanese patients with systemic sclerosis. Arthritis Rheum 1994;37:75-83.

10. Ghrénassia E, Avouac J, Khanna D, Derk CT, Distler O, Suliman YA, et al. Prevalence, correlates and outcomes of gastric antral vascular ectasia in systemic sclerosis: a EUSTAR case-control study. J Rheumatol 2014;41:99-105.
11. Nihtyanova SI, Schreiber BE, Ong VH, Rosenberg D, Moinzadeh P, Coghlan JG, et al. Prediction of pulmonary complications and long-term survival in systemic sclerosis. Arthritis Rheumatol 2014;66:1625-35.

12. Shah AA, Rosen A, Hummers L, Wigley F, Casciola-Rosen L. Close temporal relationship between onset of cancer and scleroderma in patients with RNA polymerase I/III antibodies. Arthritis Rheum 2010;62:2787-95.

13. Moinzadeh P, Fonseca C, Hellmich M, Shah AA, Chighizola C, Denton CP, et al. Association of anti-RNA polymerase III autoantibodies and cancer in scleroderma. Arthritis Res Ther 2014;16:R53.

14. Bonifazi M, Tramacere I, Pomponio G, Gabrielli B, Avvedimento EV, La Vecchia C, et al. Systemic sclerosis (scleroderma) and cancer risk: systematic review and meta-analysis of observational studies. Rheumatology 2013;52:143-54.

15. Roumm AD, Medsger TA Jr. Cancer and systemic sclerosis. An epidemiologic study. Arthritis Rheum 1985;28:1336-40.

16. Launay D, Le Berre R, Hatron PY, Peyrat JP, Hachulla E, Devulder $\mathrm{B}$, et al. Association between systemic sclerosis and breast cancer: eight new cases and review of the literature. Clin Rheumatol 2004;23:516-22.

17. Shah AA, Hummers LK, Casciola-Rosen L, Visvanathan K, Rosen A, Wigley FM. Examination of autoantibody status and clinical features that associate with cancer risk and cancer-associated scleroderma. Arthritis Rheumatol 2015;67:1053-61.

18. Joseph CG, Darrah E, Shah AA, Skora AD, Casciola-Rosen LA, Wigley FM, et al. Association of the autoimmune disease scleroderma with an immunologic response to cancer. Science 2014;343:152-7.

19. Lazzaroni MG, Cavazzana I, Colombo E, Dobrota R, Hernandez J, Hesselstrand R, et al. Malignancies in patients with anti-RNA polymerase III antibodies and systemic sclerosis: analysis of the EUSTAR cohort and possible recommendations for screening. J Rheumatol 2017;44:639-47.

20. Trallero-Araguás E, Rodrigo-Pendás JÁ, Selva-O'Callaghan A, Martínez-Gómez X, Bosch X, Labrador-Horrillo M, et al. Usefulness of anti-p155 autoantibody for diagnosing cancer-associated dermatomyositis: a systematic review and meta-analysis. Arthritis Rheum 2012;64:523-32.

J Rheumatol 2017;44:550-2; doi:10.3899/jrheum.170037 